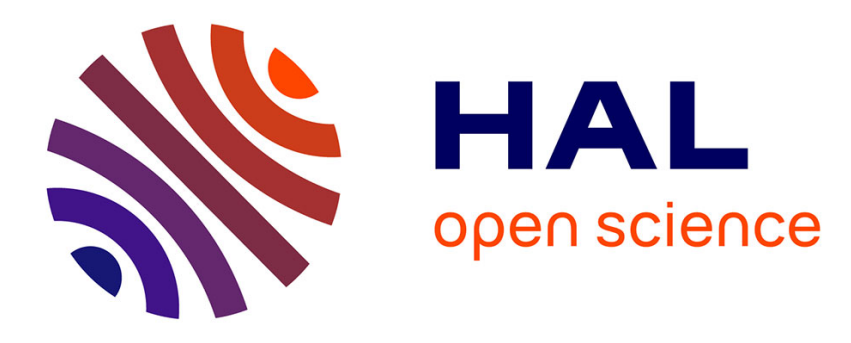

\title{
Dynamic Modeling and Stability Analysis of Mechanical Systems with Time-Varying Topologies \\ Yu Wang
}

\section{To cite this version:}

Yu Wang. Dynamic Modeling and Stability Analysis of Mechanical Systems with Time-Varying

Topologies. Journal of Mechanical Design, 1993, 115 (4), pp.808. 10.1115/1.2919272 . hal-01970469

\section{HAL Id: hal-01970469 \\ https://hal.science/hal-01970469}

Submitted on 5 Jan 2019

HAL is a multi-disciplinary open access archive for the deposit and dissemination of scientific research documents, whether they are published or not. The documents may come from teaching and research institutions in France or abroad, or from public or private research centers.
L'archive ouverte pluridisciplinaire HAL, est destinée au dépôt et à la diffusion de documents scientifiques de niveau recherche, publiés ou non, émanant des établissements d'enseignement et de recherche français ou étrangers, des laboratoires publics ou privés. 


\title{
Dynamic Modeling and Stability Analysis of Mechanical Systems with Time-Varying Topologies
}

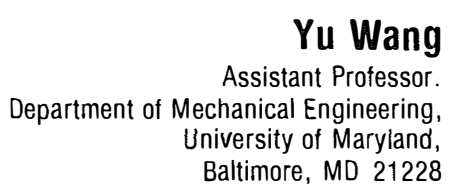

\begin{abstract}
A model is developed for analyzing mechanical systems with a pair of bodies with topological changes in their kinematic constraints. It is built upon the concept of a Poincaré map rather than following the traditional methods of differential equations. The model provides a set of well-defined and naturally-discrete equations of motion and is capable of giving physical insights of dynamic characteristics of deadbeat convergence of multiple collisions and periodic or chaotic responses. The development of a dynamic model and a local stability analysis are presented.
\end{abstract}

\section{Introduction}

Topological changes in a mechanical system often occur when kinematic constraints of the system vary with time. The changes are bound to produce jump discontinuities in the motions of bodies in the system, leaving to impulsive reaction and control forces. Examples can be found in the fields of robotics and machine dynamics. Clearances in machine joints and linkages may lead to highly nonlinear or chaotic processes. For a robotic manipulation system for object acquisition, a robot usually interacts through physical contact with an object being manipulated. The object is free to move in a certain range, before being fully controlled by the robot. In both cases the dynamic system being considered undergoes changes in its degrees of freedom, often accompanied by changes in its topology. This paper is concerned with dynamic characteristics of systems with time-varying topologies. Particularly, it focuses on the dynamics of repeated collisions in a system of two planar objects, one of which is under active control.

Since kinematic constraints of a mechanical system generally couple dynamics of its individual bodies, a topological change in the constraints causes changes in the dynamic equations of motion. The dynamic equations are generally of the following form

$$
\begin{gathered}
M \ddot{q}=f(q, \dot{q}, t)+\phi_{q}^{T}(q) \lambda \\
\phi(q, t) \geq 0
\end{gathered}
$$

where $q=\left[q_{1}, q_{2}, \ldots, q_{n}\right]^{T}$ consists of $n$ generalized coordinates and $\lambda$ is the Lagrange multiplier vector. The inequality equations describe the kinematic constraints and the differential equations are of second order. Exact closed form solutions for these equations cannot be found, except for simple systems. Hence, numerical methods must be employed.

Investigations of these systems have generally used so called "fitting" methods, in which the equations of motion are explicitly expressed between times at which discontinuous events, usually collisions, occur. The discontinuities are treated as boundary conditions to fit into the continuous equations of motion to account for the changes (Wehage and Haug, 1982; Haug, Wu, and Yang, 1986). These methods require two tasks, automatically to predict the constraint changes and numerically to integrate the differential equations (Gilmore and Cipra, 1987). Unfortunately, both tasks have many difficulties and the numerical computation is time-consuming. Furthermore, these methods can only be used to show the system dynamics for a specific initial condition. They are not able to anticipate the general dynamic characteristics of the system such as stability and regions of stability in a state space.

For systems with small relative distance between the bodies of constraint change, specific methods have been developed to analyze system dynamics (Haines, 1980). An impact pair model was used to determine specific design parameters of linkages with clearance and intermittent motion mechanisms (Dubowsky and Freudenstein, 1971; Lee and Wang, 1983). It is also used to show dynamic response of systems of harmonic excitation (Guckenheimer and Holmes, 1983; Heiman, Bajaj, and Sherman, 1988). However, as opposed to a general analysis, these methods are intended for their particular applications.

A general approach presented in Zhuravlev (1978) has also used the impact damper model. It uses a technique of nonsmooth transformation of generalized coordinates to eliminate the discontinuities in the velocities of bodies with constraint changes. The approach is suited for ideal conservative systems and for some special dissipative systems. It has been expressed in the canonical forms and applied to the analysis of motion of a solid of smooth boundary bouncing on a frictionless plane (Ivanov and Markeev, 1984). It is demonstrated that the method is effective to show general properties of system dynamics, such as stability of harmonic and subharmonic motions. Unfortunately, it is difficult to generalize this approach to general mechanical systems with damping and friction, since the energy conservation principle essentially underlines the nonsmooth transformation technique used in the method. In an asymptotic analysis presented in Nagaev (1971), the dynamic description is focused on the events of the discontinuities and equations 


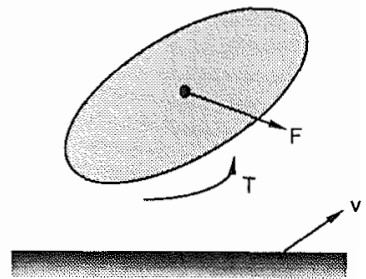

Fig. 1 The physical system of an impact pair

of motion are formulated as return maps of the modern theory of dynamical systems. However, the method is applicable to approximate asymptotic behavior only. Both approaches utilize a key property originally observed by Appell (1909) concerning changes of system momentum. The approach presented in this paper follows the same observation.

Of particular interest and concern of the present work is the dynamic study of systems in which the topological changes are due to physical contact and collision among a pair of bodies. One reason attributing to this is the need for a general method which could overcome the problem of discontinuity in representing the system dynamics and is efficient for numerical computation. Another motivation for the effort is the implication of the method for applications in the field of robotics. Many robotic manipulation tasks require physical interaction of a robot end-effector and the manipulated object. In the presence of collision, the system intermittently changes its characteristics in response to the actions of the robot. These changes result in difficulties to predict the motions of the object and the collisions raise a large bandwidth of the dynamics to be observed by a sensory-driven feedback control system (Buhler, Koditschek, and Kindlmann, 1989). A more general method is needed for planning strategies to control the system dynamics. This is essentially important for challenging robotic tasks requiring catching, throwing, and juggling. Such a method is also of general interest in the practice of automatic design of mechanical part feeders and assembly systems (Boothroyd, Redford, and Murch, 1972; Mason, 1986; Peshkin and Sanderson, 1988).

The purpose of the investigation is to develop a method capable of elucidating the motion characteristics that are due to time-varying topologies. It is hoped that the result of the investigation would provide not only a general dynamic model and efficient computational schemes, but also a deeper understanding of the dynamic characteristics. In the author's opinion, the deeper physical insight has not been well reported in the literature and is much needed for system analysis and simulation.

\section{The Model of Physical System}

The mechanical system to be studied is shown in Fig. 1, which consists of two bodies: a body with strictly convex shape moving in the vertical plane under applied force $F$ and torque $T$, and a massive plane plate with a specified vertical displacement. The plate is in theory equivalent to a solid half-space. The model is called an impact pair. It is a simplified version of many typical mechanical connections and robotic manipulation systems. Because of its simplicity, it has been taken as one of the most frequently used basic models for the investigation of mechanical systems with clearances (Haines, 1980). In spite of its simplicity, the model exhibits the typical behavior which is found in systems containing time-varying kinematic constraints. By studying this model, we shall present a new approach which would shed light on the overall system behavior and gain more physical insight into features of system dynamics. We study the system without contact friction first. Then we discuss the generalization of our approach to frictional systems.

The moving body of mass, $m$, is subject to applied forces (e.g., gravity) and comes into contact with the massive object. In the classical theory of rigid body mechanics, its linear and angular velocities undergo jump discontinuities upon colliding the massive body. Energy loss resulting from any impact is modeled by the coefficient of restitution, $e$, so that the component of relative veiocity along the contact normal ${ }^{1}$ before and after impact are related by

$$
v^{+}=-e v^{-}
$$

This is known as Newton's law.

The physical contacts may repeat many times, leading to a finite or an infinite number of collisions. With a contact the system admits one topology; without a contact it exhibits a different topology. The topology varies as a sequence of collisions take place. We derive analytical equations to describe the system dynamics in the following section.

\section{Discrete Time Formulation}

In order to characterize the system dynamics it is important to recognize that the topological changes may cease at a moment in a period of time of interest. The object stops bouncing relative to the massive object and the two objects remain continuous contact afterwards. When such a situation occurs, the object retains a continuous motion and the velocity discontinuities no longer exist. Hence, the system dynamics can be described by differential equations with algebraic constraints and studied using the methods developed for this class of systems (Gear and Petzold, 1984). We refer to the phase of motion before the qualitative change as the intermittent phase, and to the phase after as continuous phase.

Therefore, it is desirable that a method for systems with topological changes is not only able to provide a well-suited characterization of motions with multiple collisions, but is also able to identify the conditions for a convergence of the topological changes. This is of great practical interest in control and planning of robotic manipulation where initially a sequence of collisions are often inevitable but are desired to be eliminated in the course of manipulation. The goal of this section is to develop such a method.

3.1 An Example: A Dropped Tennis Ball. Before carrying out our approach, let's examine a simple example. Consider a system where a tennis ball is dropped onto a floor. The example appears trivial and may be found in any dynamics textbook; however, it provides a nice illustration of the features of interest. The tennis ball loses mechanical energy at every collision with the floor. At collision $n$, the impact is governed by Newton's law $v_{n}^{+}=-e v_{n}^{-}$where $v_{n}$ is the vertical velocity of the tennis ball at the collision, the superscripts - and + denote the instants just before and just after the collision, and $e$ is the coefficient of restitution. At the next collision $(n+$ $1)$, the velocity of the ball is given as $v_{n+1}^{-}=-v_{n}^{+}$, since mechanical energy is conserved between collisions. Hence, we obtain a difference equation for the consecutive collisions:

$$
v_{n+1}^{-}=e v_{n}^{-}
$$

and the elapsed time between the two successive collisions is $\tau_{n}=-2 e v_{n}^{-} / g$ where $g$ is the gravity acceleration. These equations show that the velocity exponentially decreases as the number of bounces increases, and the process of bouncing ceases after a finite time when the number of bounces $n$ approaches infinity. The total elapsed time $T$ from first impact to the end is the sum of the intervals,

$$
T=\sum_{n=1}^{\infty} \tau_{n}=\frac{2 e V}{(1-e) g}
$$

\footnotetext{
${ }^{1}$ If one of the contact points is a vertex, the contact normal is defined as the normal of the other body's surface. We do not consider the case of two vertices in contact.
} 


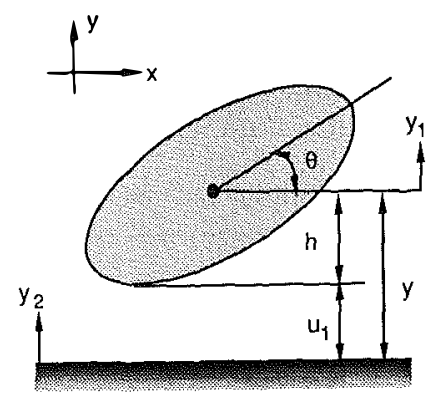

(a)

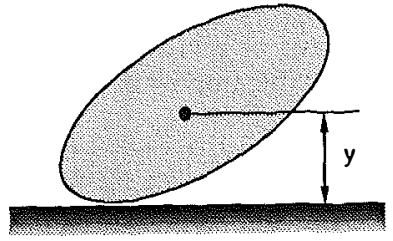

(b)
Fig. 2 The system of the object of smooth geometry and the plane surface

where $V$ is the magnitude of the initial approaching velocity $v_{1}^{-}$. After the interval $T$, the ball will be at rest on the floor.

From this example we see that the system dynamics is described by the discrete representation of the linear difference Eq. (1). The intermittent and continuous phases are also quite distinguishable. The behavior of the system in the continuous phase, for this example, is very simple and is governed by a static force balance relation. Similar to this example, the velocities of the convex body in the general system considered suffer discontinuities at collisions, foiling conventional differential equation approaches. Instead of looking for the continuous time history of motion of the system, we shall seek the system state at discrete times, more specifically at a sequence of collision events. In what follows, we shall carry out a theoretical development of the difference equation approach and show that this approach enables us to overcome the drawbacks of discontinuities.

3.2 Poincaré Maps. The discrete time formulation is obtained through the mathematical theory of dynamical systems originally proposed by Poincaré (Guckenheimer and Holmes, 1983). For an $N$ th-order continuous-time system, the theory replaces the original system with an $(N-1)$ th-order discretetime system called Poincaré map. The replacement reduces the system order and naturally bridges the gap between continuoustime and discrete-time systems.

In practice, the Poincaré map is often derived by setting a hypersurface of $(N-1)$ dimension in a state space. If the hypersurface is chosen properly, then the state trajectories will repeatedly intersect it. A Poincaré map is defined by evolution of the $(N-1)$ variables $\mathbf{x}$ at the intersection. Instead of using the continuous trajectory, one can study the system dynamics using the evolution equation

$$
\mathbf{x}(n+1)=\mathbf{f}(\mathbf{x}(n)), \quad n \text { an integer }
$$

One can also go back to the differential equations to obtain the full continuous time history of the original system if needed.

In order to construct a hypersurface to obtain a corresponding Poincaré map $\mathbf{f}$, one must have a general knowledge of the system behavior beforehand so that the state trajectories indeed cross the hypersurface. Unfortunately, there is no systematic method for this construction, although some computer programs provide helpful tools (Parker and Chua, 1987). In general, for a given system of differential equations, the function $\mathbf{f}$ is difficult to determine or it can only be determined approximately (Guckenheimer and Holmes, 1983). For our system, however, we can obtain an exact function $\mathbf{f}$, relying on the insight of system behavior. Note that a collision takes place when the two bodies come into a contact and that the bodies will move apart from each other after the collision, provided the convexity that we assumed. If the collision repeatedly occurs, the evolution of collision events could be used to characterize system dynamics. If the collisions cease to exist, the bodies either keep in constant contact or are separated,

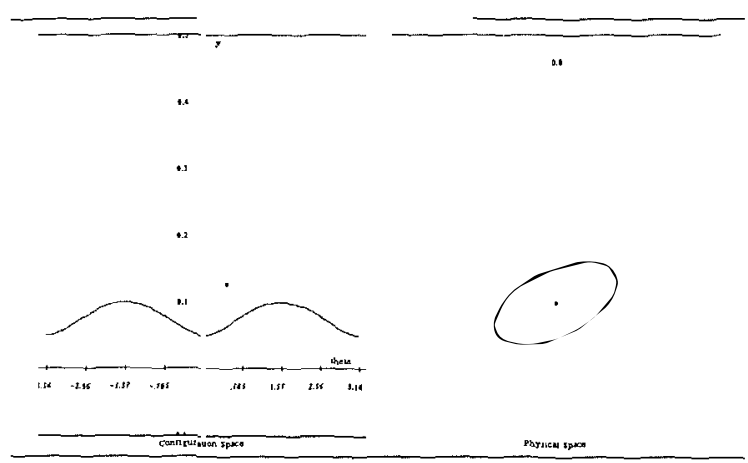

Fig. 3 An example Poincaré surface

eliminating the changes in system topology. In this case, the Poincaré map for the evolution no longer exists. Therefore, by studying the existence of the Poincaré map, we are able to obtain the conditions for qualitative changes in system dynamics. Hence, the Poincaré map is a natural choice.

3.3 Quasi-Coordinates and Velocity Discontinuities. In order to seek the state of system at a sequence of collisions, we need to express the geometric conditions of collision explicitly. We will limit ourselves to the case of single point contact between the object and the plane surface to avoid the difficulties of multiple point contact. The assumption is satisfied if the object has a strictly convex shape. The geometric condition for collision can be obtained through a quasi-coordinate $u_{1}$ which is the minimum distance between a pair of points on the geometric boundaries of the object and the plane surface, shown in Fig. 2. Note that these points are not fixed relative to the objects themselves if the bouncing object is in motion. This coordinate explicitly determines whether or not the object is in contact with the surface. When they are in contact, $u_{1}=0$; otherwise $u_{1}>0$. In the $(x, y)$ coordinate system embedded in the plane surface, the quasi-coordinate is defined by

$$
u_{1}(t)=y(t)-h(\theta(t))
$$

where, for the case of the plane surface, $y$ is the coordinate of the center of mass of the object, and $h(\theta)$ is the projection of the distance from the center of mass to the point of the object boundary on the $y$ axis. Note that $h(\theta)$ is a function of the orientation $\theta$ of the object relative to the surface only, and is independent of the displacements of the object in both $x$ and $y$ directions in this case.

The coordinate $u_{1}$ can be directly used for defining the Poincaré map as $u_{1}=0$ defines the hypersurface

$$
y-h(\theta)=0
$$

For a system shown in Fig. 3, the hypersurface is plotted in the space of coordinates $(\theta, y)$. This space is also called the configuration space as it was introduced by Lozano-Perez (1983) in the domain of motion planning for robotics. In fact, the hypersurface defined here is the configuration space boundary of the two bodies. Conceptually, the relative motion of the bodies is described in the configuration space by the motion of a point, which represents their relative kinematic configurations, relatively bouncing over the configuration space boundary. This provides a different point of view to look at the time-varying topologies.

We must also take into account the velocity discontinuities. For the rigid-body model (Section 2), the velocity discontinuities take place instantaneously and the object displacements remain unchanged during a collision. In other words, in the state space these discontinuities always occur when a trajectory intersects the hypersurface, and the state jumps from one point 


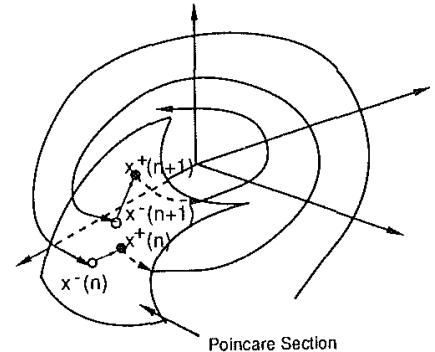

Fig. 4 Velocity jumps on the Poincaré hypersurface

to another point both on the hypersurface. This leaves two sets of state of intersection on the hypersurface: one for precollision $\left(t_{n}^{-}, \mathbf{x}\left(t_{n}^{-}\right)\right)$and the other for post-collision $\left(t_{n}^{+}, \mathbf{x}\left(t_{n}^{+}\right)\right)$, with $n$ being an integer and $\mathbf{x}$ as independent state variable vector, shown in Fig. 4. Therefore, two different Poincaré maps can be defined: (1) $\mathbf{f}^{-}$governs the discrete trajectory of pre-collision state variables

$$
\mathbf{x}^{-}(n+1)=\mathbf{f}^{-}\left(\mathbf{x}^{-}(n)\right)
$$

where $\mathbf{x}^{-}$is an augmented pre-collision state vector, possibly including variable $t_{n}$, and $(2) \mathbf{f}^{+}$of post-collision state variables

$$
\mathbf{x}^{+}(n+1)=\mathbf{f}^{+}\left(\mathbf{x}^{+}(n)\right)
$$

In this paper we arbitrarily choose the post-collision map, and the set of state vector and the Poincaré map are simply denoted as $\mathbf{x}$ and $\mathbf{f}$ unless they are particularly specified otherwise.

The concept of quasi-coordinate is well known for its special properties in dynamic analysis (Appell, 1909; Neimark and Fufaev, 1972). The time derivative of a quasi-coordinate is usually a linear function of the derivatives of generalized coordinates of Lagrange. Our choice of the quasi-coordinate $u_{1}$ has one particular advantage. It allows us to directly apply Newton's law of impact to determine the velocity discontinuity in $\dot{u}_{1}$ as

$$
\dot{u}_{1}\left(t^{+}\right)=-e \dot{u}_{1}\left(t^{-}\right)
$$

where $e$ is the coefficient of the restitution (Section 2) and $\dot{u}_{1}$ is given as

$$
\dot{u}_{1}(t)=\dot{y}(t)-h^{\prime}(\theta) \dot{\theta}(t) \quad\left(h^{\prime}=d h / d \theta\right)
$$

If we assume smooth geometric boundaries of the objects, then $\dot{u}_{1}(t)$, which is also called quasi-velocity, has the same order of continuity as $\dot{y}(t)$ and $\theta(t)$.

In addition to the quasi-coordinate $u_{1}$, we take the angular orientation of the object $\theta$ as the second independent coordinate. For the frictionless system, there is no tangential impact force, and the collision has no changes in relative motion in the tangential direction. Therefore, we need to express an additional velocity jump in $\dot{\theta}$. In the frictionless case, its expression is given as (Goldsmith, 1960),

$$
\dot{\theta}\left(t^{+}\right)-\dot{\theta}\left(t^{-}\right)=-\frac{h^{\prime}(\theta)}{h^{\prime 2}(\theta)+\rho^{2}}\left(\dot{u}_{1}\left(t^{+}\right)-\dot{u}_{1}\left(t^{-}\right)\right)
$$

Recall that for our system the Poincaré map is a function f to express the state variables right after the $n+1$ collision, in terms of their values right after the previous collision $n$. This evolution can be decomposed as two parts: a mapping $\mathbf{f}_{1}$ from the post-collision state at $t_{n}^{+}$to the next pre-collision state at $t_{n+1}^{-}$and then a mapping $\mathbf{f}_{2}$ from the pre-collision state to the post-collision state at $t_{n+1}^{+}$, namely,

$$
\mathbf{f}=\mathbf{f}_{2} \bullet \mathbf{f}_{1}
$$

using the notation for a composite function. The function $\mathbf{f}_{2}$ in fact represents the evaluation of the velocity discontinuities at collision $n+1$, which have been described above. Function $\mathbf{f}_{1}$ remains to be derived.
3.4 Difference-Algebraic Equations. The system dynamic equations in the relative coordinate system $(y, \theta)$ are given as

$$
\begin{gathered}
m \ddot{y}(t)=m\left(\ddot{y}_{1}(t)-\ddot{y}_{2}(t)\right)=F_{y}(t)-m \ddot{y}_{2}(t)+f_{y}(t) \\
m \rho^{2} \ddot{\theta}(t)=T(t)-h^{\prime}(\theta) f_{y}(t)
\end{gathered}
$$

where $f_{y}$ is the impulsive force at $y$ direction, $F_{y}$ is the component of acting force in $y$ direction, $T$ is the acting torque, and $y_{1}$ and $y_{2}$ are the absolute displacements of the object and the plane surface, separately. Note that the impulsive force $f_{y}$ exists only when an impact occurs. When there is no impact, $f_{y}=0$. Since we assume the second object of plane boundary to have a relative large mass, $y_{2}$ and $\dot{y}_{2}$ are considered to be continuous at a collision. We also have the following kinematic relationships:

$$
\begin{gathered}
\ddot{u}_{1}(t)=\ddot{y}(t)-h^{\prime} \ddot{\theta}(t)-h^{\prime \prime} \dot{\theta}^{2}(t) \\
\dot{y}(t)=\dot{u}_{1}(t)+h^{\prime}(\theta) \dot{\theta}(t)
\end{gathered}
$$

We now examine the function $\mathbf{f}_{1}$, which is obtained by integrating system equations from $t_{n}^{+}$to $t_{n+1}^{-}$. Let's consider the motion of body at time $t$ between two successive collisions at $t_{n}$ and $t_{n+1}$. The displacement and velocity of the coordinates $u_{1}$ and $\theta$ are given as, taking those at $t_{n}^{+}$as initial conditions,

$$
\begin{gathered}
\dot{u}_{1}(t)=\dot{u}_{1}\left(t_{n}^{+}\right)+\int_{t_{n}^{+}}^{t} \ddot{u}_{1}(t) d t \\
u_{1}(t)=u_{1}\left(t_{n}^{+}\right)+\int_{t_{n}^{+}}^{t} \dot{u}_{1}(t) d t \\
\dot{\theta}(t)=\dot{\theta}\left(t_{n}^{+}\right)+\int_{t_{n}^{+}}^{t} \ddot{\theta}(t) d t \\
\theta(t)=\theta\left(t_{n}^{+}\right)+\int_{t_{n}^{+}}^{t} \dot{\theta}(t) d t
\end{gathered}
$$

After substituting the above kinematic and dynamic equations into them, the expressions become

$$
\begin{aligned}
\dot{u}_{1}(t)= & \dot{y}(t)-h^{\prime}(\theta(t)) \dot{\theta}(t) \\
= & \dot{y}\left(t_{n}^{+}\right)+\int_{t_{n}}^{t}\left(Q_{1}(t)-\ddot{y}_{2}(t)\right) d t-h^{\prime}(\theta(t)) \\
& \times\left(\dot{\theta}\left(t_{n}^{+}\right)+\int_{t_{n}}^{t} Q_{2}(t) d t\right) \\
= & \dot{u}_{1}\left(t_{n}^{+}\right)-\left(h^{\prime}(\theta(t))-h^{\prime}\left(\theta\left(t_{n}^{+}\right)\right)\right) \dot{\theta}\left(t_{n}^{+}\right) \\
& +\int_{t_{n}^{+}}^{t} Q_{1}(t) d t-\left(\dot{y}_{2}(t)-\dot{y}_{2}\left(t_{n}^{+}\right)\right)-h^{\prime}(\theta(t)) \\
& \times \int_{t_{n}^{+}}^{t} Q_{2}(t) d t \\
u_{1}(t)= & y(t)-h(\theta(t)) \\
& +\int_{t_{n}^{+}}^{t}\left(\int_{t_{n}^{+}}^{t}\left(Q_{1}(t)-\ddot{y}_{2}(t)\right) d t\right) d t-h(\theta(t)) \\
& y\left(t_{n}^{+}\right)+\dot{y}\left(t_{n}^{+}\right)\left(t-t_{n}^{+}\right) \\
&
\end{aligned}
$$




$$
\begin{gathered}
=u_{1}\left(t_{n}^{+}\right)+\left(\dot{u}_{1}\left(t_{n}^{+}\right)+h^{\prime}\left(\theta\left(t_{n}^{+}\right)\right) \dot{\theta}\left(t_{n}^{+}\right)\right)\left(t-t_{n}^{+}\right) \\
+\int_{t_{n}^{+}}^{t}\left(\int_{t_{n}^{+}}^{t} Q_{1}(t) d t\right) d t-\left(y_{2}(t)-y_{2}\left(t_{n}^{+}\right)\right. \\
\left.-\dot{y}_{2}\left(t_{n}^{+}\right)\left(t-t_{n}^{+}\right)\right)-\left(h(\theta(t))-h\left(\theta\left(t_{n}^{+}\right)\right)\right) \\
\dot{\theta}(t)=\dot{\theta}\left(t_{n}^{+}\right)+\int_{t_{n}^{+}}^{t} Q_{2}(t) d t \\
=\dot{\theta}\left(t_{n}^{+}\right)+\int_{t_{n}^{+}}^{t} Q_{2}(t) d t \\
\theta(t)=\theta\left(t_{n}^{+}\right)+\dot{\theta}\left(t_{n}^{+}\right)\left(t-t_{n}^{+}\right)+\int_{t_{n}^{+}}^{t}\left(\int_{t_{n}^{+}}^{t} Q_{2}(t) d t\right) d t \\
=\theta\left(t_{n}^{+}\right)+\dot{\theta}\left(t_{n}^{+}\right)\left(t-t_{n}^{+}\right)+\int_{t_{n}^{+}}^{t}\left(\int_{t_{n}^{+}}^{t} Q_{2}(t) d t\right) d t
\end{gathered}
$$

where

$$
\begin{aligned}
& Q_{1}(t)=\frac{F_{y}(t)}{m} \\
& Q_{2}(t)=\frac{T(t)}{m \rho^{2}}
\end{aligned}
$$

We evaluate these equations at $t=t_{n+1}^{-}$and, for clarity, we drop superscripts + and - for the continuous quantities, $t$, $u_{1}, \theta, y_{2}$, and $\dot{y}_{2}$. We then obtain

$$
\begin{aligned}
& \dot{u}_{1}\left(t_{n+1}^{-}\right)=\dot{u}_{1}\left(t_{n}^{+}\right)-\left(h^{\prime}\left(\theta\left(t_{n+1}\right)\right)\right. \\
& \left.-h^{\prime}\left(\theta\left(t_{n}\right)\right)\right) \dot{\theta}\left(t_{n}^{+}\right)+\int_{t_{n}}^{t_{n+1}} Q_{1}(t) d t \\
& -\left(\dot{y}_{2}\left(t_{n+1}\right)-\dot{y}_{2}\left(t_{n}\right)\right)-h^{\prime}\left(\theta\left(t_{n+1}\right)\right) \int_{t_{n}}^{t_{n+1}} Q_{2}(t) d t \\
& u_{1}\left(t_{n+1}\right)=u_{1}\left(t_{n}\right)+\left(\dot{u}_{1}\left(t_{n}^{+}\right)+h^{\prime}\left(\theta\left(t_{n}\right)\right) \dot{\theta}\left(t_{n}^{+}\right)\right)\left(t_{n+1}-t_{n}\right) \\
& +\int_{t_{n}}^{t_{n+1}} \int_{t_{n}}^{t} Q_{1}(s) d s d t-\left(y_{2}\left(t_{n+1}\right)-y_{2}\left(t_{n}\right)\right. \\
& \left.-\dot{y}_{2}\left(t_{n}\right)\left(t_{n+1}-t_{n}\right)\right)-\left(h\left(\theta\left(t_{n+1}\right)\right)-h\left(\theta\left(t_{n}\right)\right)\right) \\
& \dot{\theta}\left(t_{n+1}^{-}\right)=\dot{\theta}\left(t_{n}^{+}\right)+\int_{t_{n}}^{t_{n+1}} Q_{2}(t) d t \\
& \theta\left(t_{n+1}\right)=\theta\left(t_{n}\right)+\dot{\theta}\left(t_{n}^{+}\right)\left(t_{n+1}-t_{n}\right)+\int_{t_{n}}^{t_{n+1}} \int_{t_{n}}^{t} Q_{2}(s) d s d t
\end{aligned}
$$

Up to this point, we have accomplished the derivations for the function $\mathbf{f}_{1}$, expressing the state at $t_{n+1}^{-}$in terms of the state at $t_{n}^{+}$. Using the expressions for the velocity discontinuities in Eqs. (4) and (6), the entire Poincaré function is ready to be completed. Noting that at the collisions the kinematic constraint requires that

$$
u_{1}\left(t_{n}\right)=u_{1}\left(t_{n+1}\right)=0
$$

and using a convention that variable $n$ representing the time instant right after collision $n$ at $t_{n}^{+}$and that $\tau(n)$ representing the time interval between the collisions $n$ and $n+1$, we obtain the system equations in terms of the variables $u_{1}, \dot{u}_{1}, \theta, \theta, t$, and $\tau$, for the case $e>0$,

$$
\begin{gathered}
u_{1}(n+1)=0 \\
\dot{u}_{1}(n+1)=-e\left\{\dot{u}_{1}(n)-\left[h^{\prime}(\theta(n+1))-h^{\prime}(\theta(n))\right] \theta(n)\right. \\
+\int_{t(n)}^{t(n+1)} Q_{1}(t) d t-\left[\dot{y}_{2}(t(n+1))-\dot{y}_{2}(t(n))\right] \\
\left.-h^{\prime}(\theta(n+1)) \int_{t(n)}^{t(n+1)} Q_{2}(t) d t\right\} \\
\theta(n+1)=\theta(n)+\dot{\theta}(n) \tau(n)+\int_{t(n)}^{t(n+1)} \int_{t(n)}^{t} Q_{2}(s) d s d t \\
\dot{\theta}(n+1)=\dot{\theta}(n)-\frac{1+e}{e} \frac{h^{\prime}(\theta(n+1))}{\rho^{2}+h^{\prime 2}(\theta(n+1))} \dot{u}_{1}(n+1) \\
t(n+1)=t(n)+\tau(n)
\end{gathered}
$$

and

$$
\begin{aligned}
& \int_{t(n)}^{t(n+1)} \int_{t(n)}^{t} Q_{1}(s) d s d t+\dot{y}_{2}(t(n)) \tau(n) \\
& -\left[y_{2}(t(n+1))-y_{2}(t(n))\right]+\left[\dot{u}_{1}(n)+h^{\prime}(\theta(n)) \dot{\theta}(n)\right] \tau(n) \\
& -[h(\theta(n+1))-h(\theta(n))]=0 \quad
\end{aligned}
$$

The difference Eqs. (24)-(28) can be rewritten into explicit difference equations. Moreover, the system is nonautonomous since the variable $t(n)$ is explicitly in the equations. We may ignore variable $u_{1}(n)$ since Eq. (24) always defines the Poincaré section and $u_{1}(n)=0$. Instead of using $u_{1}(n)$, we introduce $t(n)$ as a state variable. Then the equations can be rearranged to become an autonomous system of the difference-algebraic equations of the augmented state variable $\mathbf{x}(n)=$ $\left[t(n) \dot{u}_{1}(n) \theta(n) \dot{\theta}(n)\right]^{T}$ in a vector form:

$$
\begin{gathered}
\mathbf{x}(n+1)=\mathbf{f}(\mathbf{x}(n), \tau(n), \mathbf{g}(\mathbf{x}(n), \tau(n))) \\
\phi(\mathbf{x}(n), \tau(n), \mathbf{g}(\mathbf{x}(n), \tau(n)))=0
\end{gathered}
$$

where $\tau(n)$ is the elapsed time from $t(n)$ to $t(n+1)$ and $\mathbf{g}$ represents the applied forces on the object and the absolute motions of the plane surface. The vector Eq. (30) represents the difference Eqs. (25)-(28), while the scalar Eq. (31) defines the algebraic Eq. (29).

Functions $f$ and $\phi$ are continuous and differentiable. Hence, through the application of the Poincare's method, we have obtained a well-defined discrete formulation. This development will enable us to apply conventional techniques to analyze the system dynamic characteristics without any problems of discontinuity.

\section{Stability Analysis}

The difference-algebraic equations derived above are highly nonlinear, and it is difficult to find exact solutions except for simple systems. However, the behavior of solutions for arbitrary initial conditions as $n \rightarrow \infty$ can be characterized by the local stability of equilibrium states (also called fixed points), and the global regions of their stability. The local property shall provide some insights into the dynamic features of the system, whereas the global analysis shows the overall behavior of the nonlinear systems. In this section, we discuss the fixed points and their stability. The global analysis and the numerical simulation are presented in Wang (1993).

The characteristics of dynamic behavior is related to the 


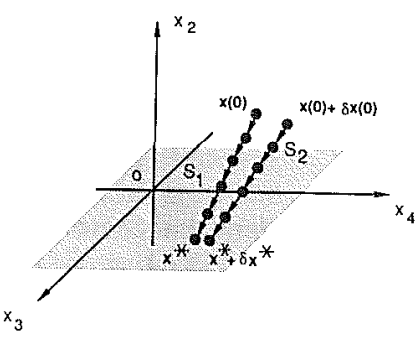

Fig. 5 Discrete trajectories and fixed points

properties of function $\mathbf{g}$ which represents the applied forces and the motion of the massive plane object. We shall refer to it as the driving function. Stability of system response to the driving function is the major concern of this section. This is of practical significance, as discussed in Wang (1989b) to the development of control strategies for manipulation tasks in some dynamic environments. We study the general case of dissipative system $(0<e<1)$. For a perfectly plastic system $(e=0)$, the problem becomes trivial and after the first collision the object usually continuously contacts the surface; for a perfectly elastic system $(e=1)$, the collisions become conservative and the system exhibits special dynamic behavior (Wang, 1989a).

4.1 Equilibrium State and Fixed Point. Our differencealgebraic equations are in the semi-explicit form with the explicit first order vector difference Eq. (30) and the scalar Eq. (31). The system is analogous to the system of differentialalgebraic equations of continuous time model discussed in Gear and Petzold (1984). We may regard the variable $\tau(n)$ as an intermediate variable since it does not possess an evolution, and the algebraic Eq. (31) as a constraint on the intermediate variable. Thus, the system is determinate because the number of intermediate variables is equal to the number of constraints (Gear and Petzold, 1984).

A fixed point $\mathbf{x}^{*}$ satisfies

$$
\begin{gathered}
\mathbf{x}^{*}=\mathbf{f}\left(\mathbf{x}^{*}, \tau^{*}, \mathbf{g}\left(\mathbf{x}^{*}, \tau^{*}\right)\right) \\
\phi\left(\mathbf{x}^{*}, \tau^{*}, \mathbf{g}\left(\mathbf{x}^{*}, \tau^{*}\right)\right)=0
\end{gathered}
$$

and the associated time $t^{*}=t(\infty)$ as $n \rightarrow \infty$ could be either finite or infinite, depending on whether $\Sigma_{n} \tau(n)$ converges.

Let's at this moment exclude possible periodic equilibrium states, for which both harmonic and subharmonic motions must be considered. Then we find the fixed points for this system

$$
\begin{aligned}
& x_{1}^{*}=t^{*}=\text { constant } \\
& x_{2}^{*}=\dot{u}_{1}^{*}=0 \\
& x_{3}^{*}=\theta^{*}=\text { constant } \\
& x_{4}^{*}=\dot{\theta}^{*}=\text { constant }
\end{aligned}
$$

and correspondingly

$$
\tau^{*}=0
$$

These fixed points are not isolated. In a sub-state space of variables $x_{2}, x_{3}$, and $x_{4}$, the fixed points are connected and consist of the plane $x_{2}=0$ (Fig. 5). Every point on the plane is a state of equilibrium, representing the state of zero relative normal velocity at the contact point and of arbitrary relative angular position and velocity.

4.2 Steady-State Solutions and Their Local Stability. Having found the fixed point, we now study its local stability characteristics. Here, stability or instability will be taken in the sense of Lyapunov. The local stability of a fixed point $\mathbf{x}^{*}$ is represented by that of the linearized system equations. Let $\mathbf{z}=\left[\begin{array}{llll}z_{1} & z_{2} & z_{3} & z_{4}\end{array}\right]^{T}$ be the perturbation for the state vector, and $\zeta$ be the perturbation for variable $\tau(n)$, respectively,

$$
\begin{aligned}
& \mathbf{x}(n)=\mathbf{x}^{*}+\mathbf{z}(n) \\
& \tau(n)=\tau^{*}+\zeta(n)
\end{aligned}
$$

When discussing the local stability, the perturbation variables $\mathbf{z}$ and $\zeta$ are assumed to be small, and when substituting these into Eqs. (30) and (31) and expanding their right-hand side functions around the fixed point $\mathbf{x}^{*}$, and $\tau^{*}$, the nonlinear terms in the Taylor series expansion are usually neglected. Thus, we obtain a system of linear equations:

$$
\begin{gathered}
\mathbf{z}(n+1)=\mathbf{A z}(n)+\mathbf{b} \zeta(n) \\
\mathbf{c}^{T} \mathbf{z}(n)+d \zeta(n)=0
\end{gathered}
$$

where the Jacobian matrix A has components

$$
\mathbf{A}=\begin{array}{llll}
1 & 0 & 0 & 0 \\
0 & e & 0 & 0 \\
0 & \alpha & 1 & 0 \\
0 & \beta & 0 & 1
\end{array}
$$

with

$$
\alpha=\frac{-2 x_{4}^{*}}{Q_{1}\left(t^{*}\right)-\ddot{y}_{2}\left(t^{*}\right)-h^{\prime}\left(x_{3}^{*}\right) Q_{2}\left(t^{*}\right)-h^{\prime \prime}\left(x_{3}^{*}\right) x_{4}^{* 2}}
$$

and

$$
\begin{gathered}
\beta=-(1+e) \frac{h^{\prime}\left(x_{3}^{*}\right)}{\rho^{2}+h^{\prime 2}\left(x_{3}^{*}\right)} \\
-Q_{1}\left(t^{*}\right)-\ddot{y}_{2}\left(t^{*}\right)-h^{\prime}\left(x_{3}^{*}\right) Q_{2}\left(t^{*}\right)-h^{\prime \prime}\left(x_{3}^{*}\right) x_{4}^{* 2} \\
\mathbf{b}^{T}=\left[\begin{array}{llll}
1 & 0 & 0 & 0
\end{array}\right] \\
\mathbf{c}^{T}=\left[\begin{array}{llll}
0 & 1 & 0 & 0
\end{array}\right]
\end{gathered}
$$

if

$$
d=\frac{1}{2}\left[Q_{1}\left(t^{*}\right)-\ddot{y}_{2}\left(t^{*}\right)-h^{\prime}\left(x_{3}^{*}\right) Q_{2}\left(t^{*}\right)-h^{\prime \prime}\left(x_{3}^{*}\right) x_{4}^{*}\right] \neq 0
$$

The stability of the solution $\mathbf{z}(n)=0$ for the linear system is completely determined by the matrix $\mathbf{A}$. The stability criteria are discussed in many books on difference equations, for example, (Bernussou, 1977). Here, we summarize some of relevant results.

(1) The solution for the linear system is asymptotically stable if and only if all the eigenvalues of $\mathbf{A}$ are of absolute values less than one.

(2) The solution is not stable if one of the eigenvalues has absolute value greater than one.

(3) If there are eigenvalues whose absolute values are equal to one and all other eigenvalues have absolute values less than one, then the linear system is stable when the eigenvalues of absolute values equal to one have independent eigenvectors.

The third case in which at least one eigenvalue has absolute value equal to one is referred to as the critical case. For the critical case, the local stability for the linearized system cannot be directly carried over to its original nonlinear system. It usually requires higher order terms in the Taylor series to determine the local stability of the nonlinear system (Bernussou, 1977). However, if the fixed point is not isolated, then we have a degenerate case, for which the stability characteristics for the linearized system may be used to evaluate the stability of the fixed point for the original nonlinear system.

Eigenvalues of $\mathbf{A}$ are found by evaluating

$$
|\lambda \mathbf{I}-\mathbf{A}|=0
$$

The four eigenvalues are real 


$$
\lambda_{1}=e \text { and } \lambda_{2}=\lambda_{3}=\lambda_{4}=1
$$

and three of them are repeated eigenvalues with unit magnitude, but the multiple eigenvalue has linearly independent eigenvectors. Together with the first independent eigenvector associated with the eigenvalue $\lambda_{1}=e$, these eigenvectors are

$$
\begin{aligned}
& \mathbf{v}_{1}=\left[\begin{array}{llll}
0 & 1-e & -\alpha & -\beta
\end{array}\right]^{T} \\
& \mathbf{v}_{2}=\left[\begin{array}{llll}
1 & 0 & 0 & 0
\end{array}\right]^{T} \\
& \mathrm{v}_{3}=\left[\begin{array}{llll}
0 & 0 & 1 & 0
\end{array}\right]^{T} \\
& \mathbf{v}_{4}=\left[\begin{array}{llll}
0 & 0 & 0 & 1
\end{array}\right]^{T}
\end{aligned}
$$

Noting that the linearized system is in the critical case and that the fixed point is not isolated, we may directly find the solutions for the linearized system by carefully examining the structure of the linear equations:

$$
\begin{gathered}
z_{1}(n)=\frac{2}{(1-e) W^{*}} C e^{n} \\
z_{2}(n)=C e^{n} \\
z_{3}(n)=\frac{2}{1-e} \frac{x_{4}^{*}}{W^{*}} C e^{n} \\
z_{4}(n)=\frac{1+e}{1-e} \frac{h^{\prime}\left(x_{3}^{*}\right)}{\rho^{2}+h^{\prime 2}\left(x_{3}^{*}\right)} C e^{n} \\
\zeta(n)=-\frac{2}{W^{*}} C e^{n}
\end{gathered}
$$

where

$$
W^{*}=Q_{1}\left(t^{*}\right)-\ddot{y}_{2}\left(t^{*}\right)-h^{\prime}\left(x_{3}^{*}\right) Q_{2}\left(t^{*}\right)-h^{\prime \prime}\left(x_{3}^{*}\right) x_{4}^{* 2} \neq 0
$$

and $C$ is a constant to be determined from the initial conditions.

In these solutions, the magnitudes of the variables $\mathbf{z}(n)$ and $\zeta(n)$ exponentially decay as $n \rightarrow \infty$. The $\operatorname{sum} \Sigma_{n}^{\infty} \zeta(n)$ of the intervals $\zeta(n)$ converges to a finite value. This means that in a finite time interval the discrete trajectory approaches the fixed point and the repeated collisions come to an end, although an infinite number of collisions take place. Then a continuous contact begins. These properties resemble those of the tennis ball system shown in Eq. (1), even though in addition to the bouncing motion $z_{2}$ this system has angular motions $z_{3}$ and $z_{4}$.

Since the first eigenvalue satisfies $\left|\lambda_{1}\right|=e<1$ and the multiple eigenvalue of magnitude equal to one has linearly independent eigenvectors, the connected fixed point is the degenerate critical case. In a strict sense, the system is not asymptotically stable. For a trajectory $S_{1}$ in the neighborhood of the fixed point, let us perturb the initial state $\mathbf{x}(0)$ slightly to its neighbor $\mathbf{x}(0)+\delta \mathbf{x}(0)$. Then the new trajectory $S_{2}$ starting at the neighbor will not converge to the fixed point $x^{*}$ corresponding to the original trajectory. Instead, it converges to another fixed point $\mathbf{x}^{*}+\delta \mathbf{x}^{*}$ in its vicinity (Fig. 5). The perturbation $\delta \mathbf{x}(0)$ in the initial state leads to the discrepancies $\delta \mathbf{x}^{*}$ in the final state variables except for $x_{2}^{*}$ which approaches zero as $n \rightarrow \infty$ despite the perturbation.

The degeneracy of the critical fixed point is a result of the physical nature of the system. The system is of second order, but the energy storage and dissipation elements are not completely represented if we treat it as a mass-damper-spring system. In fact, there is no spring-like element to resist the motion of the $u_{1}$ coordinate. This leads to an extra degeneracy that is not reflected in the difference equations since the variable $u_{1}(n)$ is ignored in the discrete formulation. Similarly, in coordinate $\theta$ both spring-like and damper-like elements do not exist. The system motion is essentially free in that coordinate, resulting in two more degrees of degeneracy. If we remove the only dissipative element by making the system perfectly elastic, then the fixed points and their local stability will change dra- matically, causing the system to behave in a totally different manner (Wang, 1989a).

In the solutions for the linear system (36)-(40), $z_{2}(n)$ is the component of the relative velocity at the contact point along the common normal. It is evaluated at the moment of postcollision, and must be non-negative, as is the elapsed time $\zeta(n)$. Therefore, it is evident that these solutions are feasible only for a negative $W^{*}$ and a positive constant $C$. This means that the conditions for successive collisions require that the object accelerate towards the surface in the course of the collisions, since $W^{*}$ represents the relative acceleration of the object toward the plane surface. This condition is expressed as

$$
W^{*}=Q_{1}\left(t^{*}\right)-\ddot{y}_{2}\left(t^{*}\right)-h^{\prime}\left(x_{3}^{*}\right) Q_{2}\left(t^{*}\right)-h^{\prime \prime}\left(x_{3}^{*}\right) x_{4}^{*}<0
$$

This condition has an intuitive meaning. If the object is placed on the surface of the massive object and remains in contact, then the object may have a rolling motion of angular velocity $\dot{\theta}(t)$. In order to maintain the contact, it is necessary that (Wang, 1989a)

$$
Q_{1}(t)-\ddot{y}_{2}(t)-h^{\prime}[\theta(t)] Q_{2}(t)-h^{\prime \prime}[\theta(t)] \dot{\theta}^{2}(t)<0
$$

This suggests that the condition for local-stable convergence of a sequence of collisions to a continuous contact is identical to the condition for continuous rolling without separation. Of course, this result is not surprising.

When $W^{*}>0$, the solutions of the linear equations give either negative time intervals $\tau(n)$ or negative rebounding velocities $z_{2}(n)$. The solutions for the latter attempt to represent the motion of the object coming from the inside of the massive half-space and moving towards the open half space. Once the object touches the boundary, it is bounced into the solid again. This is mirror-symmetric to the realistic situation that the object can move only in the open half-space. The symmetry of the solutions reflects the fact that the equations only represent geometric boundaries, without a notion of solid occupied volume. This problem, called viability, can be resolved by simply discarding solutions of nonphysical significance. For general systems, special care may be required to accomplish this (Heiman et al., 1988).

Moreover, there is a special case that we must take care of. When $W^{*}=0$, the second order terms in the Taylor series expansions are needed to determine stability. The following condition is required

$$
\dot{W}\left(t^{*}\right)>0
$$

However, this condition guarantees that the collision process ceases as $n \rightarrow \infty$, but the object will not continue to keep contact with the surface afterwards. In fact, contact will break again just when the successive collisions are about to come to an end. This is because the relative acceleration $W(t)$ between the object and the surface becomes positive after the moment $t^{*}$, and the plane surface slows relative to the object. The contact cannot pull the object, and the object will leave the contact surface right after the moment.

In summary, there are three possibilities at an equilibrium state, depending on the value of the relative acceleration $W^{*}$ : (1) The equilibrium is unstable $\left(W^{*}>0\right)$ and it can never be reached; (2) The equilibrium is stable $\left(W^{*}<0\right)$. In this case, the bouncing motion becomes deadbeat in a finite time interval as an infinite number of collisions converge. The stability is called deadbeat stability; (3) The equilibrium is marginally stable $\left(W^{*}=0\right.$ and $\left.W^{*}>0\right)$. These three cases are illustrated in Fig. 6.

4.3 Rate of Convergence. The rate of convergence of a stable equilibrium can be illustrated geometrically. For the noncritical case $\left(W^{*}<0\right)$, the asymptotic solutions are dominated by the linearized system solutions or the first order 


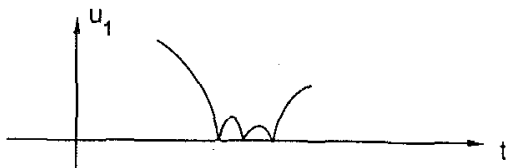

(a)

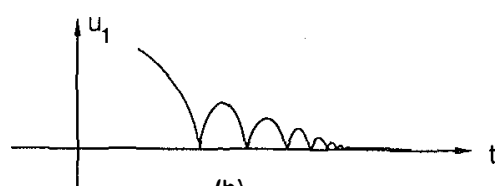

(b)

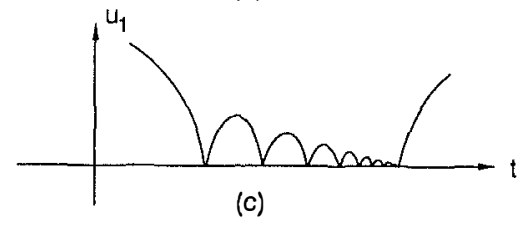

Fig. 6 Possible cases of repeated collisions

expansions. Let us examine the elapsed time $\tau(n)$. We have found its solution, Eq. (40)

$$
\tau(n)=C e^{n}
$$

with a constant $C$. For a given initial value $\tau(0)$, there corresponds a unique sequence $\tau(0), \tau(1), \tau(2), \ldots$. This sequence can be represented by an iteration process defined by

$$
\tau(n+1)=e \tau(n)
$$

which is described graphically using rectangular coordinates with axes $\tau(n)$ and $\tau(n+1)$ by two straight lines defined by $\tau(n+1)=\tau(n)$ and $\tau(n+1)=e \tau(n)$ as shown in Fig. $7(\boldsymbol{a})$. From the initial value $\tau(0)$ the sequence eventually approaches the origin, and the iteration process converges with the magnitude of the variable $\tau(n)$ reduced by $e$ for each iteration.

For the critical case $\left(W^{*}=0\right)$, the approximated system must involve the second-order expansions because the first order term $W^{*}$ vanishes. Generally it is cumbersome to derive the second-order or higher-order expansions (Levy and Lessman, 1961). In an asymptotic analysis for a closed related problem, Nagaev (1971) proposed the following form of series expansions

$$
\begin{aligned}
& x_{i}(n)=\sum_{m=0}^{\infty}{ }^{m} x_{i}(n) \epsilon^{m} \text { for } i=1,3,4 \\
& x_{2}(n)=\sum_{m=2}^{\infty}{ }^{m} x_{2}(n) \epsilon^{m} \\
& \tau(n)=\sum_{m=0}^{\infty}{ }^{m} \tau(n) \epsilon^{m}
\end{aligned}
$$

and an addition series expansion for $\dot{x}_{2}(n)$

$$
\dot{x}_{2}(n)=\sum_{m=1}^{\infty}{ }^{m} \dot{x}_{2}(n) \epsilon^{m}
$$

where $\epsilon=A \lambda^{n}$ and $A$ and $\lambda$ are constants.

Similarly, after substituting these expressions into the difference-algebraic equations and equating coefficients of like powers of $\epsilon$ on both sides of the equations, we again obtain a set of first order difference equations for each order $m$ of $\epsilon^{m}$. However, unlike the noncritical case, the equations are nonlinear and their solutions are polynomials of variable $\epsilon=A \lambda^{n}$ instead, where the constant $\lambda$ is a positive root of the equation

$$
\lambda^{3}+2 \lambda^{2}-2 e \lambda-e=0
$$

The equation has a single positive root whose value is always

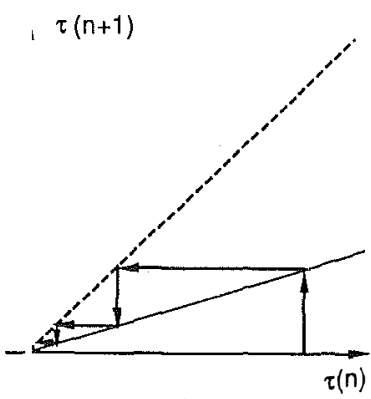

(a)

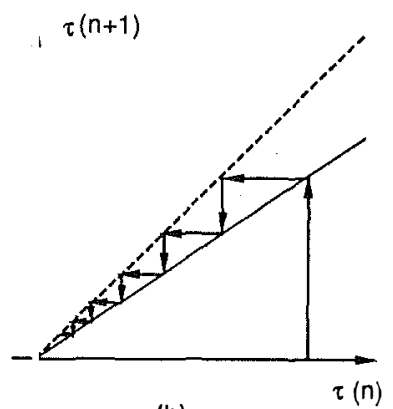

(b)
Fig. 7 Convergence of variable $\tau(n)$

greater than the coefficient of restitution $e$ and less than 1 ( $e$ $<\lambda<1)$. The solution for $\tau(n)$ is

$$
\tau(n)=D \lambda^{n} \quad(\lambda>e)
$$

where $D$ is a constant. The iteration process

$$
\tau(n+1)=\lambda \tau(n)
$$

also converges. However, the rate of convergence is slower than that of the noncritical case since $\lambda$ is greater than $e$, Fig. $7(b)$. We omit the fine details of the analysis and the reader is referred to Levy and Lessman (1961) and Nagaev (1971) for further discussions.

\section{Systems with Contact Friction}

In the presence of contact friction between the bodies, the Poincare hypersurface is defined the same as in the frictionless case, but the Poincaré map becomes more complicated. During a collision a frictional impulse $P_{x}$ is generated along the common tangent of the contact points. If we adopt Coulomb's law of dry friction, the magnitude of the frictional impulse is related to the magnitude of normal impulse $P_{y}$ by a coefficient of friction $\mu$ as follows

$$
\left|P_{x}\right| \leq \mu P_{y}
$$

The inequality relationship requires further conditions on contact modes of impact to determine the exact value of the frictional impulse. The derivations for these conditions are fairly complicated and lengthy, as presented in Wang (1989a); Wang and Mason (1992). Here, we only outline the results. A contact mode of impact describes how the tangential relative motion of the contact points changes during the collision. In the case of Coulomb's model, the contact points may slide relative to each other, or stick, or stick and then reversely slide. Accordingly, there are three contact modes: sliding, sticking, and reversed sliding. Conditions for these contacts modes are the functions of the pre-collision system state variables. In general, the state space is divided into three regions, called contact regions, each corresponding to a contact mode. Once the contact mode is identified, a unique solution to the impulse is determined (Wang and Mason, 1992).

One may recall that the Poincaré map consists of two parts: $f_{1}$ and $f_{2}$, Eq. (7). The former describes the motion of the object free from the contact constraint and maps a post-collision state to a pre-collision state of the next collision. The function $\mathbf{f}_{1}$ is unchanged for the frictional system, except for an additional set of equations for the motion in the $x$ coordinate. The second function $\mathbf{f}_{2}$ describes the changes in velocities at a collision and maps the pre-collision state into the post-collision state. These velocity discontinuities in the frictional system are substantially different from those for the frictionless system derived in the previous section. Their analytical expressions for each contact mode are different. Therefore, the final difference-algebraic equations are of the form 


$$
\begin{aligned}
& \mathbf{x}(n+1)= \\
& \left(\begin{array}{ll}
\mathbf{f}_{s}(\mathbf{x}(n), \tau(n), \mathbf{g}(\mathbf{x}(n), \tau(n))) & \text { for sliding contact } \\
\mathbf{f}_{c}(\mathbf{x}(n), \tau(n), \mathbf{g}(\mathbf{x}(n), \tau(n))) & \text { for sticking contact } \\
\mathbf{f}_{r}(\mathbf{x}(n), \tau(n), \mathbf{g}(\mathbf{x}(n), \tau(n))) & \text { for reversed sliding contact }
\end{array}\right.
\end{aligned}
$$

and

$$
\phi(\mathbf{x}(n), \tau(n), \mathbf{g}(\mathbf{x}(n), \tau(n)))=0
$$

where the vector $\mathbf{x}$ is the augmented state variable vector as before but including $x(n)$ and $\dot{x}(n)$. The exact expressions for these functions are described in Wang (1989a) but not presented here due to space limit.

The mapping function $\mathbf{f}$ is defined by the functions $\mathbf{f}_{s}, \mathbf{f}_{c}$, and $\mathbf{f}_{r}$ for each contact mode respectively and $\mathbf{f}$ is continuous but piecewise differentiable with respect to the state variables. In other words, the function has continuous derivatives inside each contact region (i.e., for each contact mode), and the derivatives approach finite values as any point on the boundary of each region is approached from its interior; but the values are different if the boundary point is approached from interiors of different regions which share the boundary.

The piecewise differentiability result in more complexity for the local and global stability analysis. If the fixed point is in the interior of a contact region then the method of local analysis presented in Section 4 can be directly applied, and we would have similar results to those for the frictionless system. However, if the fixed point is on a boundary between contact regions, we must determine in which contact region to perform the local analysis. This depends on the interior of the contact region from which the discrete trajectory approaches the fixed point. Consequently, the results for that contact region should be applied. In the global analysis and numerical simulation (Wang, 1993), these factors do not cause any problems if the contact regions are borne in mind and carefully coded in numerical programs.

\section{Conclusions}

Mechanical systems with a pair of bodies may establish a sequence of collisions. When the bodies contact and separate, the topology of system changes drastically. A model of impact pair has been developed to describe the system dynamics by a discrete time representation. A function of point mapping or a Poincaré map is defined to explicitly describe an evolution process of the multiple collisions. The resulting differencealgebraic equations are well-defined and suited for dynamic analysis and numerical simulation. A local stability analysis has shown physical insight to the deadbeat convergence of multiple collisions and the characteristics of the topological changes. The aspects of global analysis and numerical computation are the subjects of another study (Wang, 1993).

This paper is confined in the domain of planar bodies with one being represented by a massive plane surface. The presented approach, however, can be applied to a broader class of systems such as a system of objects both with curved boundaries as discussed in (Wang, 1989a). We have emphasized the deadbeat behavior of a series of collisions in the presence of material inelasticity and contact friction. It is possible that the system would exhibit periodical or chaotic responses to an excitation of the massive body. This will be discussed elsewhere.

\section{Acknowledgments}

I would like to thank Matt Mason and Randy Brost for their discussions in the development and presentation of this paper.
This work was supported in part by the System Development Foundation and by the National Science Foundation under grant DMC-8520475.

\section{References}

Appell, P. E., 1909, Traite de Mecanique Rationelle, Vol. 2, Gauthier-Villars, Paris.

Bernussou, J., 1977, Point Mapping Stability, Pergamon Press, New York.

Boothroyd, G., Redford, A. H., and Murch, C. P., 1972, "Statistical Distributions of Natural Resting Aspects of Parts for Automatic Handling," Manufacturing Engineering, Transactions of Society of Manufacturing Automation, Vol. 1, pp. 93-105.

Buhler, M., Koditschek, D. E., and Kindlmann, P. J., 1989, "Planning and Control of Robotic Juggling Tasks," The Fifth International Symposium on Robotics Research, Tokyo, Japan.

Dubowsky, S., and Freudenstein, F., 1971, "Dynamic Analysis of Mechanical Systems With Clearances, Part 1: Formulation of Dynamic Model," ASME Journal of Engineering for Industry, Vol. 93, pp. 305-309.

Gear, C. W., and Petzold, L. R., 1984, "Ode Methods for the Solution of Differential/Algebraic Systems," SIAM Journal on Numerical Analysis, Vol. 21, No. 4, pp. 716-728.

Gilmore, B. J., and Cipra, R. J., 1987, "Simulation of Planar Dynamic Mechanical Systems With Changing Topologies: Part 1-Characterization and Prediction of the Kinematic Constraint Changes; Part 2-Implementation Strategy and Simulation Results for Example Dynamic Systems," ASME Advances in Design Automation - 1987, S. S. Rao, ed., pp. 369-388.

Goldsmith, W., 1960, Impact: The Theory and Physical Behavior of Colliding Solids, Edward Arnold Ltd., London.

Guckenheimer, J., and Holmes, P., 1983, Nonlinear Oscillations, Dynamica Systems, and Bifurcations of Vector Fields, Springer-Verlag, New York. Also, Second Printing, 1986.

Haines, R. S., 1980, "Survey: 2-dimensional Motion and Impact at Revolute Joints," Mechanism and Machine Theory, Vol. 15, pp. 361-370.

Haug, E. J., Wu, S. C., and Yang, S. M., 1986, "Dynamics of Mechanical Systems With Coulomb Friction, Stiction, Impact and Constraint Addition Deletion-Part 1, Theory," Mechanism and Machine Theory, Vol. 21, No. 5 pp. 401-406.

Heiman, M. S., Bajaj, A. K., and Sherman, P. J., 1988, "Periodic Motions and Bifurcations in Dynamics of an Inclined Impact Pair," Journal of Sound and Vibration, Vol. 124, No. 1, pp. 55-78.

Ivanov, A. P., and Markeev, A. P., 1984, "The Dynamics of Systems with Unilateral Constraints," Journal of Applied Mathematics and Mechanics, Vol. 48 , No. 4, pp. 448-451.

Lee, T. W., and Wang, A. C., 1983, "On the Dynamics of Intermittent Motion Mechanisms-Part 1, Dynamic Model and Response," ASME JourNAL of Mechanisms, Transmissions, and Automation in Design, Vol. 105, pp. 534-540.

Levy, H., and Lessman, F., 1961, Finite Difference Equations, Macmillan; New York.

Lozano-Perez, T., 1983, "Spatial Planning: A Configuration Space Approach," IEEE Transactions on Computers, Vol. C-32, No. 2, pp. 108-120.

Mason, M. T., 1986, "Mechanics and Planning of Manipulator Pushing Operations," International Journal of Robotics Research, Vol. 5, No. 3, pp. 53-71

Nagaev, R. F., 1971, "The General Problem of Quasi-Plastic Impact," Me chanics of Solids, Vol. 6, No. 3, pp. 83-90

Neimark, I. I., and Fufaev, N. A., 1972, Dynamics of Nonholonomic Systems, American Mathematical Society, Providence, RI.

Parker, T. S., and Chua, L. O., 1987, "Insite-A Software Toolkit for the Analysis of Nonlinear Dynamical Systems," Proceedings of the IEEE, Vol. 75, No. 8, pp. 1081-1089.

Peshkin, M. A., and Sanderson, A. C., 1988, "The Motion of a Pushed, Sliding Workpiece," IEEE Journal of Robotics and Automation, Vol. 4, No. 6, pp. 569-598.

Wang, Y., 1989a, Dynamic Analysis and Simulation of Mechanical Systems With Intermittent Constraints, Ph.D. thesis, Department of Mechanical Engi neering, Carnegie Mellon University.

Wang, Y., 1989b, "Dynamics and Planning of Collisions in Robotic Manipulation," IEEE International Conference on Robotics and Automation, Scottsdale, AZ, pp. 478-483.

Wang, Y., 1993, "Global Analysis and Simulation of Mechanical Systems with Time-Varying Topologies," ASME Journal of Mechanical Design, In press.

Wang, Y., and Mason, M. T., 1992, "Two-dimensional Rigid Body Collisions With Friction," ASME Journal of Applied Mechanics, Vol. 59, pp. 635-642.

Wehage, R. A., and Haug, E. J., 1982, "Dynamic Analysis of Mechanical Systems With Intermittent Motion," ASME Journal of Mechanical Design Vol. 104, pp. 778-784

Zhuravlev, V. F., 1978, "Equations of Motion of Mechanical Systems With Ideal One-Sided Links," Journal of Applied Mathematics and Mechanics, Vol. 42, No. 5, pp. 839-847. 